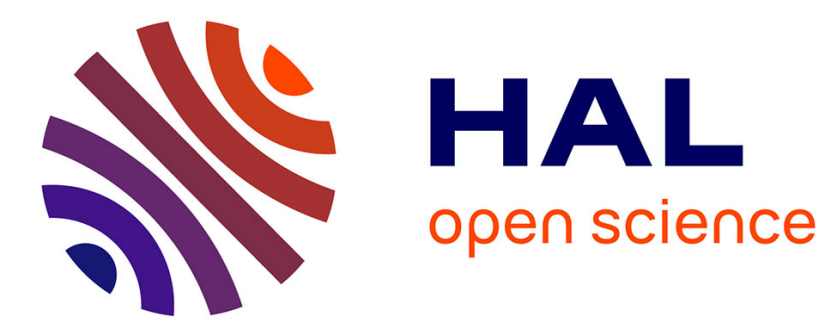

\title{
Optimal Control of Interacting Systems with DNSS Property: The Case of Illicit Drug Use
}

\author{
I. Zeiler, J.P. Caulkins, G. Tragler
}

\section{To cite this version:}

I. Zeiler, J.P. Caulkins, G. Tragler. Optimal Control of Interacting Systems with DNSS Property: The Case of Illicit Drug Use. Journal of Economic Behavior and Organization, 2011, 78 (1-2), pp.60. 10.1016/j.jebo.2010.12.008 . hal-00978258

\section{HAL Id: hal-00978258 https://hal.science/hal-00978258}

Submitted on 14 Apr 2014

HAL is a multi-disciplinary open access archive for the deposit and dissemination of scientific research documents, whether they are published or not. The documents may come from teaching and research institutions in France or abroad, or from public or private research centers.
L'archive ouverte pluridisciplinaire HAL, est destinée au dépôt et à la diffusion de documents scientifiques de niveau recherche, publiés ou non, émanant des établissements d'enseignement et de recherche français ou étrangers, des laboratoires publics ou privés. 


\section{Accepted Manuscript}

Title: Optimal Control of Interacting Systems with DNSS

Property: The Case of Illicit Drug Use

Authors: I. Zeiler, J.P. Caulkins, G. Tragler

PII: $\quad$ S0167-2681(11)00007-2

DOI: $\quad$ doi:10.1016/j.jebo.2010.12.008

Reference: $\quad$ JEBO 2653

To appear in: Journal of Economic Behavior \& Organization

Received date: $\quad 11-8-2009$

Revised date: $\quad 29-12-2010$

Accepted date: $\quad 29-12-2010$

Please cite this article as: Zeiler, I., Caulkins, J.P., Tragler, G., Optimal Control of Interacting Systems with DNSS Property: The Case of Illicit Drug Use, Journal of Economic Behavior and Organization (2008), doi:10.1016/j.jebo.2010.12.008

This is a PDF file of an unedited manuscript that has been accepted for publication. As a service to our customers we are providing this early version of the manuscript. The manuscript will undergo copyediting, typesetting, and review of the resulting proof before it is published in its final form. Please note that during the production process errors may be discovered which could affect the content, and all legal disclaimers that apply to the journal pertain. 


\title{
Optimal Control of Interacting Systems with DNSS Property: The Case of Illicit Drug Use
}

\author{
I. Zeiler ${ }^{*, a}$, J.P. Caulkins ${ }^{\mathrm{b}}$, G. Tragler $^{\mathrm{a}}$ \\ ${ }^{a}$ Department of Mathematical Methods in Economics, Vienna University of Technology, \\ Argentinierstrasse 8/105-4, A-1040 Vienna, Austria \\ ${ }^{b}$ Carnegie Mellon University Qatar Campus, Adelaide Campus, Heinz College's School of \\ Public Policy $\&$ Management School of Information Systems $\&$ Management, \\ 5000 Forbes Ave. Pittsburgh, PA 15213
}

\begin{abstract}
In this paper we generalize a one-dimensional optimal control problem with DNSS property to a two-dimensional optimal control problem. This is done by taking the direct product of the model with itself, i.e. we combine two similar system dynamics under a joint objective functional that is separable in both states and controls. This framework can be applied to the construction of various optimal control problems, such as optimal marketing of related products, optimal growth of separate but interacting economies, or optimal control of two related capital stocks.

We study such a system for a particular case drawn from the domain of drug control. The main result of this paper is that in this domain even a modest amount of interaction can sometimes make a very big difference. Hence, drawing conclusions by simplifying the real world into two independent, one-dimensional models may be problematic.

Methodologically the combination of two systems with DNSS property leads to a fascinating series of situations with multiple optimal steady states and associated threshold behavior. These instances reflect some important recent developments in optimal dynamic control theory.
\end{abstract}

Key words:

optimal control, indifference points, multiple equilibria, DNSS points, illicit

\footnotetext{
*Corresponding author

Email addresses: irmgard.zeiler@alumni.tuwien.ac.at (I. Zeiler), caulkins@cmu.edu (J.P. Caulkins), tragler@eos.tuwien.ac.at (G. Tragler)
}

Preprint submitted to Journal of Economic Behavior $\&$ Organization January 20, 2011 
drug use, interacting systems

2000 MSC: C44, C61, O40

\section{Introduction}

Optimal control models have played an important role in economic theory. Over a long period most of the models analyzed yielded a unique and stable equilibrium solution. In that situation the qualitative conclusions derived from such models are robust with respect to the initial conditions. Dechert and Nishimura (1983), Sethi (1977, 1979), and Skiba (1978) seem to be the first who presented examples of optimal control models with multiple locally optimal equilibria. Which equilibrium is optimal to converge to depends then on where one $\operatorname{starts}^{1}$; hence the initial conditions play a crucial role. Naturally, such equilibria are separated by indifference or tipping points, which are more formally known as Skiba or DNSS points (henceforth, DNSS in honor of the four seminal authors). In a one-state system, DNSS points are initial conditions at which the decision maker is indifferent between moving "right" or "left" by applying a control such that the state variable approaches either a high-level or a low-level steady state.

A classic example is the Ramsey growth model with a convex-concave production function of Skiba (1978). If a society starts with a relatively generous supply of capital, the welfare maximizing policy will involve further investment and growth toward a high-income equilibrium. In contrast, a society starting with a capital stock below the threshold level should remain in that low-capital, low-production, low-consumption domain because the long-run benefits of investment do not outweigh the short-run benefits of consumption.

Another well-known model with multiple steady states concerns marketing. Sethi (1979) analyzed a version of the contagion model for optimal advertising (Gould, 1970). He showed that when information spreads by word of mouth, rather than through a standard advertising medium, the optimal outcome may depend on the initial level of awareness about the firm.

\footnotetext{
${ }^{1}$ An early analysis of multiple equilibria and history dependence is provided by Schelling $(1967,1973)$ who assumed that the probability that an agent engages in an illegal transaction increases with the total number of agents who also engage in the transaction. This results in three equilibrium points, a clean and a dirty society separated by an unstable interior tipping point.
} 
For very low initial awareness, decaying awareness (forgetting) dominates the word of mouth transmission effects and the firm will become unknown and should go out of business. This is the low-level steady state. If initial awareness is high enough the firm should advertise at the maximum allowable level to attain a positive, high-level steady-state awareness fraction.

Besides applications in business and economics, tipping points are of great interest in economic-ecological models, e.g. Brock and Starret (2003), Mäler et al. (2003), and Wagener (2003). Other applications include fashion (Caulkins et al., 2007), crime (Feichtinger et al., 2002), and migration (Caulkins et al., 2005).

Scholars very reasonably investigate one-dimensional systems first because they are more tractable and intuition developed with one-dimensional models often carries over to more complicated models. However, a model with a single state variable is almost always a substantial simplification that omits interaction with the outside world. To take the Ramsey growth model as an example, individual nations' economies are rarely isolated. Usually there is both trade and migration across international borders, so it seems sensible to ask how if at all the insights produced by the Ramsey model might be modified if there were two economies that interacted with each other in some fashion? Likewise, one might wish to generalize Sethi's (1979) optimal advertising problem to two states, one for the firm and one for the firm's main product. Awareness of the product may then spill over to awareness of the firm and vice versa, and this spill over connects the two states by generating additional awareness in each state. Examples are a motor vehicle manufacturer and a specific car brand or a fashion company and its most known piece of clothing.

More generally one might ask what happens if one takes the "direct product" of two (or more) one-dimensional optimal control models that exhibit tipping points. Such a model, where one decision maker can control two related states, was first considered by Brock and Dechert (1983) but without any interaction between the states. So in this paper we link two otherwise independent systems, and ask what if any difference that linkage makes.

When the presence of an additional, parallel one-state system has little effect on the solution or policy prescriptions for the other one-state system, then we have some reassurance that not much was lost by simplifying the world down to a single one-state system and studying that system. On the other hand, if modest interaction with a second system greatly perturbs the solution to the original system, then the conclusions we thought we "knew" 
based on the one-state model might need to be stated with an extra caveat or expressed in conditional terms, conditional on the absence of significant interactions with parallel systems.

What we do in this paper is in some sense a proof by example that introducing modest interaction to the direct product of two isolated one-state systems can make a substantial difference in the optimal solution. The particular case investigated is drawn from the domain of drug control and the main result here is that in this domain even a modest amount of interaction seems to sometimes make a very big difference. For example, one might expect some sort of monotonicity in the optimal outcome with growing interaction. However, we show that small perturbations of the linkage of the systems can lead to a total reversal of the optimal policy. This is all the more significant when one considers that not just the magnitude of the interaction was modest, but also that the nature of the interaction belongs to a specific class. In our context the interaction is drug users counted by one state variable moving over to the other state (e.g. switching drugs). In more general terms this means that the sum of the state equations only depends directly on the current states and not at all on the presence or the extent of interaction.

The paper is structured as follows. In Section 2 we present a general modeling framework for combining two independent systems under one objective functional and introduce the particular model of illicit drug use. The optimal control problem is solved by applying Pontryagin's Minimum Principle. Section 3 discusses the substantial changes that occur to the one-dimensional system when a parallel, interacting system is introduced. We do this by presenting three different numerical illustrations of the strong impact of interaction. The paper closes in Section 4 with a discussion of the results and conclusions for the optimal control of interacting, parallel systems.

\section{The Model}

\subsection{General Modeling Framework}

We discuss two parallel non-linear, autonomous, optimal control problems with infinite planning horizon

$$
\min _{u_{A}(t)} J_{A}=\int_{0}^{\infty} e^{-r t} g_{A}\left(x_{A}(t), u_{A}(t), \rho_{A}\right) d t
$$


subject to

$$
\dot{x}_{A}(t)=f_{A}\left(x_{A}(t), u_{A}(t), \rho_{A}\right),
$$

and

$$
\min _{u_{B}(t)} J_{B}=\int_{0}^{\infty} e^{-r t} g_{B}\left(x_{B}(t), u_{B}(t), \rho_{B}\right) d t
$$

subject to

$$
\dot{x}_{B}(t)=f_{B}\left(x_{B}(t), u_{B}(t), \rho_{B}\right),
$$

where $x_{i}(t) \in X_{i} \subset \mathbb{R}$ are the state variables, $u_{i}(t) \in U_{i} \subset \mathbb{R}^{n}$ are the control or policy variables, $\rho_{i} \in \Upsilon_{i} \subset \mathbb{R}^{q_{i}}$ are $q_{i}$-dimensional parameters for $i \in\{A, B\}$. For reasons of simplification we assume identical time discount rates, $r_{A}=r_{B}=r$. We call such systems "parallel" if they are related in content such that introducing interaction is sensible in the application.

The two-dimensional optimal control problem is built by summing up and weighting the cost functionals of each optimization problem; i.e. the two-dimensional model is generated by two jointly managed one-dimensional problems. The new objective functional is separable in the cost functionals, i.e. without further interaction an optimization of the joint system would lead to the same results as optimizing each individual system. This is altered when a functional is introduced that connects $\dot{x}_{A}(t)$ and $\dot{x}_{B}(t)$ in some form.

Letting $h_{i}(\cdot), i \in\{A, B\}$, denote the flow functionals and $\sigma \in \Upsilon_{I} \subset \mathbb{R}^{s}$ an $s$-dimensional parameter that governs the extent of interaction between the systems, the resulting two-dimensional model reads as

$$
\min _{u_{A}(t), u_{B}(t)} J=\int_{0}^{\infty} e^{-r t}\left(\varpi_{A} g_{A}\left(x_{A}(t), u_{A}(t), \rho_{A}\right)+\varpi_{B} g_{B}\left(x_{B}(t), u_{B}(t), \rho_{B}\right)\right) d t
$$

subject to

$$
\begin{aligned}
& \dot{x}_{A}(t)=f_{A}\left(x_{A}(t), u_{A}(t), \rho_{A}\right)+h_{A}\left(x_{A}(t), x_{B}(t), \rho_{A}, \rho_{B}, \sigma\right), \\
& \dot{x}_{B}(t)=f_{B}\left(x_{B}(t), u_{B}(t), \rho_{B}\right)+h_{B}\left(x_{A}(t), x_{B}(t), \rho_{A}, \rho_{B}, \sigma\right),
\end{aligned}
$$

where $\left(x_{A}, x_{B}\right) \in X_{A} \times X_{B} \subset \mathbb{R}^{2}$ denote the state variables, $\left(u_{A}, u_{B}\right) \in$ $U_{A} \times U_{B} \subset \mathbb{R}^{n} \times \mathbb{R}^{n}$ the controls and $\left(\rho_{A}, \rho_{B}\right) \in \Upsilon_{A} \times \Upsilon_{B} \subset \mathbb{R}^{q_{A}} \times \mathbb{R}^{q_{B}}$ a pair of parameters. The weights, $\varpi_{A}$ and $\varpi_{B}$, reflect the decision maker's preferences.

In this paper we consider a special case for which $h_{A}(\cdot)=-h_{B}(\cdot)$ so the interaction does not directly affect the sum of the states. The added flows 
are from one state to the other and vice versa, but not into or out of the system overall.

As stand-alone (non-interacting) systems, both models (1) and (2) are assumed to manifest two locally optimal steady states that are separated by a DNSS point. If $\underline{x}$ and $\bar{x}$ are two such optimal steady states, a DNSS point, $x=x^{D N S S}$, is an initial state for which there are two distinct optimal controls, $u_{1}(t)$ and $u_{2}(t)$, and corresponding state trajectories, $x_{1}(t)$ and $x_{2}(t)$, with $x_{1}(0)=x_{2}(0)=x^{D N S S}, \lim _{t \rightarrow \infty} x_{1}(t)=\underline{x}$ and $\lim _{t \rightarrow \infty} x_{2}(t)=\bar{x}$, such that $J\left(x_{1}(t), u_{1}(t)\right)=J\left(x_{2}(t), u_{2}(t)\right)$. Hence, when starting at a DNSS point a system may be driven to either steady state while incurring equal costs.

If the states in the two-dimensional problem (3) are decoupled, i.e. $h_{i}(\cdot)=$ 0 , the DNSS property of the one-dimensional case is naturally inherited to this case. In particular the two-dimensional DNSS thresholds create a simple cross (see Brock and Dechert, 1983). In the following we want to find out what happens to the DNSS thresholds when the connecting flows are nonzero and discuss this problem by the example of interacting drug epidemics.

\subsection{The Drug Control Model}

The particular example we use to construct a two-dimensional model of type (3) is drawn from the domain of drug use and control. Optimal control theory has been harnessed for about a decade to provide crucial insights concerning how drug control policy ought to adapt over the course of a drug epidemic. In particular, previous work (e.g. Tragler et al., 2001) showed that the occurrence of DNSS thresholds is a common phenomenon that describes what is thought to hold true for drug epidemics. When control begins early, i.e. when the epidemic is still small, it is optimal to drive it down to a low level of use. It is common to refer to this as "eradication" even though the lowlevel equilibrium may not be at zero. If the size of the epidemic crosses the DNSS threshold then instead of eradication, an "accommodation" strategy is indicated that delays rather than controls the growth of the drug problem towards a high level of use.

The general idea that drug markets can be stable at either low- or highlevels of participation is borne out in data and observation. If the market is very small, for any given level of drug control (e.g. enforcement), the control effort per participant will be high and that potentially induces some to exit. Each exit shrinks the market size and further inflates the density of control per remaining participant. This can create a virtuous positive feedback loop in which that same fixed level of control can be enough to suppress the 
market, and, in a subsequent maintenance phase, an even smaller level of control may be enough to keep an eliminated market from rebounding. So "eradication" refers to a stabilization of the epidemic at such a level, where the absence of scale economics makes it relatively easy to maintain the market at that size (Kleiman, 1993). However, if the market is initially beyond some minimum threshold size, then the initial control effort per participant is low, which may give more people an incentive to enter. That lets the market expand to some high level equilibrium where an eradication strategy is not feasible or at least not optimal.

In the optimal control literature, so far only single populations of drug users have been considered. This is a simplification since most societies with a serious drug problem have substantial use of more than one substance, and/or have to deal with mobile drug users that may affect the course and costs of an epidemic in places other than their origin (see, e.g., Roxburgh et al., 2004; Rachlis et al., 2007). So, besides discussing an important question on optimal control, we introduce here for the first time an optimal control model that considers two interacting populations of drug users.

The underlying one-state one-control model is based on Tragler et al's. (2001) work on the optimal dynamic allocation of treatment and enforcement. To better suit our needs we modified the model in two ways. First, we replaced Tragler et al.'s growth function (a power function) by the more familiar logistic function that models illicit drug use as diffusion process. This better describes the common "lifecycle" for a drug in a particular society, which is minimal levels of use for an extended period before some event stimulates market growth that then leads to convex growth in initiation and, eventually, stabilization at a higher endemic level of use (Caulkins, 2008). For more on the diffusion of drug use in a population, see also Ferrence (2001). Second, we use a single control whose effect is removing users, referred to for convenience as "treatment". The price-raising control "law enforcement" is omitted for simplicity, which leads to a constant retail price. Constant prices are clearly a simplification, but it is common in drug policy analysis to assume a very high elasticity of supply and, hence, a price that varies little with prevalence, because there are few constrained factors of production. Bultmann et al. (2008a,b) discuss these modifications at length.

As in Tragler et al. (2001) the objective is to minimize the discounted sum over time of the control costs, $u(t)$, plus consumption, $p^{-\omega} A(t)$, times the social costs per unit consumed, $\kappa$. The dynamics of $A(t)$ is governed by three flows, a logistic inflow, an endogenous outflow, and a control driven 
outflow.

The impact of control spending is as in Rydell et al.'s (1996) model of drug treatment. However, we prefer to think of it more generally as any control driven outflow from drug use. For example, deterring or incarcerating users is an alternative, non-treatment mechanism for removing users. Control has diminishing returns for the usual reasons. For example, for treatment there may be "cream skimming" in which those who are easiest to treat are treated first. Hence, the rate at which users exit due to control, $\beta(A(t), u(t))$, is concave and monotonically increasing in control spending $u(t)$, i.e. assuming $A>0$, then $\beta^{\prime}>0$ and $\beta^{\prime \prime}<0$. In particular, following Rydell et al. (1996) we have

$$
\beta(A(t), u(t))=c\left(\frac{u(t)}{A(t)}\right)^{z}
$$

where $c$ is the constant measuring the average efficiency of control and $z$ the constant measuring the marginal efficiency of control with $0<z<1$ (cf. Tragler et al., 2001; Behrens et al., 2000, 2002). The endogenous outflow due to natural desistance occurs at a constant per capita rate.

Hence, we investigate a special case of the optimal control problem (1) where

$$
\begin{gathered}
g(A(t), u(t), \rho)=\kappa p^{-\omega} A(t)+u(t) \\
f(A(t), u(t), \rho)=k p^{-a} A(t)(m-A(t))-c\left(\frac{u(t)}{A(t)}\right)^{z} A(t)-\mu p^{b} A(t) .
\end{gathered}
$$

Both the state and control variables must be nonnegative. The parameter set $\rho$ at the base case values consists of

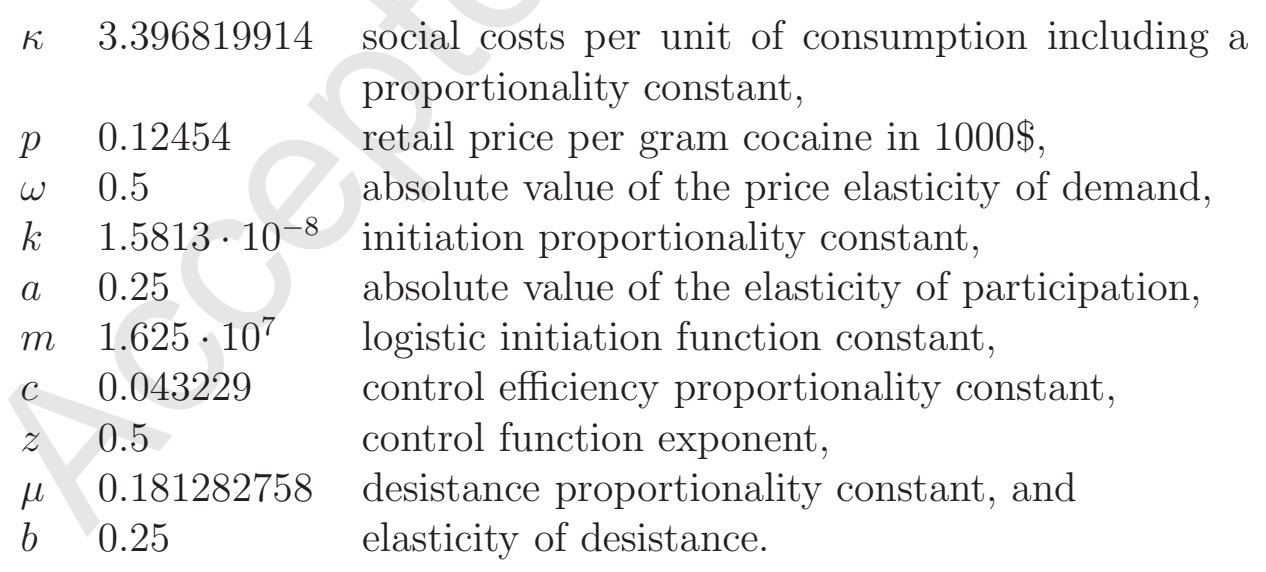

The time discount rate, $r$, is 0.04 . 
Several forms of $h(\cdot)$ are possible. Here, the flows are chosen to be linear in the state variable and independent of the control and second state; thus, $h_{A}(\cdot)=-\gamma_{A} A+\gamma_{B} B$ and $h_{B}(\cdot)=-h_{A}(\cdot)$, where $\gamma_{A}$ denotes the rate at which users leave population $A$ by migrating into $B$, and $\gamma_{B}$ the rate at which users leave population $B$ by migrating into $A$. At one moment in time an individual can only be part of one population, either $A$ or $B$. So, "migration" can be understood as physical migration from e.g. one city to another or in a wider sense as a change of primary substance of abuse.

Furthermore we assume the cost functionals to be equally weighted in the joint objective functional, hence we have $\varpi_{A}=\varpi_{B}=1$.

So when $A(t)$ and $B(t)$ denote the current numbers of users of a drug, the two-dimensional model of coupled parallel systems is given by

$$
\min _{u_{A}(t) \geq 0, u_{B}(t) \geq 0} J=\int_{0}^{\infty} e^{-r t}\left(\kappa_{A} p_{A}^{-\omega_{A}} A(t)+\kappa_{B} p_{B}^{-\omega_{B}} B(t)+u_{A}(t)+u_{B}(t)\right) d t,
$$

subject to the system dynamics

$$
\begin{aligned}
\dot{A}(t)= & k_{A} p_{A}^{-a_{A}} A(t)\left(m_{A}-A(t)\right)-c_{A}\left(\frac{u_{A}(t)}{A(t)}\right)^{z_{A}} A(t) \\
& -\mu_{A} p_{A}^{b_{A}} A(t)-\gamma_{A} A(t)+\gamma_{B} B(t) \\
\dot{B}(t)= & k_{B} p_{B}^{-a_{B}} B(t)\left(m_{B}-B(t)\right)-c_{B}\left(\frac{u_{B}(t)}{B(t)}\right)^{z_{B}} B(t) \\
& -\mu_{B} p_{B}^{b_{B}} B(t)+\gamma_{A} A(t)-\gamma_{B} B(t) .
\end{aligned}
$$

\subsection{Solution of the Optimal Control Problem}

To solve the optimal control problem (6)-(8) by applying Pontryagin's Minimum Principle (cf. Feichtinger and Hartl, 1986; Grass et al., 2008; Leonard and Long, 1992) we consider the current value Hamiltonian $\mathcal{H}=\mathcal{H}\left(A, B, \lambda_{A}, \lambda_{B}, u_{A}, u_{B}\right)$ defined by

$$
\mathcal{H}=\lambda_{0}\left(g_{A}\left(A, u_{A}, \rho_{A}\right)+g_{B}\left(B, u_{B}, \rho_{B}\right)\right)+\lambda_{A} \dot{A}+\lambda_{B} \dot{B},
$$

where $\lambda_{A}$ and $\lambda_{B}$ denote the costate variables in current value terms and $\lambda_{0} \geq 0$ is a nonnegative constant multiplier associated with the integrand of the objective function

$$
g_{A}\left(A, u_{A}, \rho_{A}\right)+g_{B}\left(B, u_{B}, \rho_{B}\right)=\kappa_{A} p_{A}^{-\omega_{A}} A+\kappa_{B} p_{B}^{-\omega_{B}} B+u_{A}+u_{B} .
$$


The time argument $t$ is omitted for brevity. If we assumed that $\lambda_{0}=0$, then the Hamiltonian minimizing condition would yield at least one $u_{i}=\infty$, $i \in\{A, B\}$, because $\mathcal{H}$ is monotonically decreasing in $u_{i}$ and there is no upper bound on the controls. That would cause the objective function (6) to be maximized which contradicts our purpose of minimizing the total societal costs. Hence, we can conclude that $\lambda_{0} \neq 0$ and may set $\lambda_{0}=1$ without loss of generality.

There must exist two non-positive multipliers $\eta_{A}$ and $\eta_{B}$ for the nonnegativity constraints on the controls such that the partial derivatives of the Lagrangian function $\mathcal{L}=\mathcal{L}\left(A, B, \lambda_{A}, \lambda_{B}, u_{A}, u_{B}, \eta_{A}, \eta_{B}\right)$ with respect to the controls are equal to zero when evaluated along an optimal path. The Lagrangian function is defined by

$$
\mathcal{L}=\mathcal{H}+\eta_{A} u_{A}+\eta_{B} u_{B}
$$

and the complementary slackness conditions

$$
\eta_{i} \leq 0 \wedge \eta_{i} u_{i}=0, \forall i \in\{A, B\}
$$

imply that as long as the optimal controls are in the interior of the feasible region, i.e. strictly positive, the Lagrangian function $\mathcal{L}$ reduces to the Hamiltonian $\mathcal{H}$ and the solutions of the constrained problem equals the unconstrained one.

So, if $\forall i \in\{A, B\}: u_{i}>0$, the necessary ${ }^{2}$ optimality condition on the controls is to minimize the Hamiltonian subject to the constraints at any instant of time,

$$
u_{i}^{\star}=\arg \min _{u_{i}} \mathcal{H} .
$$

Using the convexity of the Hamiltonian with respect to the controls, which is proven by the positive definiteness of the Hessian matrix,

$\frac{\partial^{2} \mathcal{H}}{\partial u_{i} \partial u_{j}}=\left(\begin{array}{cc}-\frac{1}{A} c_{A}\left(\frac{u_{A}}{A}\right)^{z_{A}-2}\left(z_{A}-1\right) z_{A} \lambda_{A} & 0 \\ 0 & -\frac{1}{B} c_{B}\left(\frac{u_{B}}{B}\right)^{z_{B}-2}\left(z_{B}-1\right) z_{B} \lambda_{B}\end{array}\right)$,

$i, j \in\{A, B\}$, we can derive

$$
\begin{aligned}
& u_{A}^{\star}=A\left(c_{A} z_{A} \lambda_{A}\right)^{\frac{1}{1-z_{A}}}, \\
& u_{B}^{\star}=B\left(c_{B} z_{B} \lambda_{B}\right)^{\frac{1}{1-z_{B}}},
\end{aligned}
$$

\footnotetext{
${ }^{2}$ Sufficiency holds if the Hamiltonian is jointly convex in both the states and the controls.
} 
for the controls by setting $\mathcal{H}_{u_{i}}=0$. If any $u_{i}=0, i \in\{A, B\}$, (12) yields $\eta_{i} \leq 0$, where an explicit form for $\eta_{i}$ can be derived from $\mathcal{L}_{u_{i}}=0$.

Furthermore, the costate equations are given by

$$
\dot{\lambda}_{A}=r \lambda_{A}-\mathcal{H}_{A} \text { and } \dot{\lambda}_{B}=r \lambda_{B}-\mathcal{H}_{B}
$$

The limiting transversality conditions for the costates $\lambda_{A}$ and $\lambda_{B}$ are

$$
\lim _{t \rightarrow \infty} \mathbf{e}^{-r t} \lambda_{A}(t)=0 \text { and } \lim _{t \rightarrow \infty} \mathbf{e}^{-r t} \lambda_{B}(t)=0
$$

and hold if states and costates approach a stable state. After substituting for the controls, the state and costate equations form the canonical system, and their two-dimensional stable manifolds satisfy the necessary optimality conditions provided by the Minimum Principle.

\section{Results}

The goal of this section is to discuss how the optimal solution of decoupled parallel systems changes when interaction is introduced. Before discussing this interesting question we present the solution of two identically parameterized, jointly managed systems. This acts as base case for the analysis of coupled cases, i.e. it is an instance to which the later results can be compared. For the baseline parametrization we use the values of Bultmann et al. (2008b) (see Section 2.2) that roughly describe the current U.S. cocaine epidemic and yield in the one-state one-control model two optimal steady states, $\underline{A}$ and $\bar{A}$, that are separated by a DNSS point, $A_{D N S S}$. We then present three different illustrations for the strong impact of interaction. In every case the separate state's internal dynamics is similar, so these are modest departures from the noninteracting case. In particular, two illustrations involve identical systems; the third has asymmetry in that state $B$ cannot be controlled and does not impose any costs directly (e.g. represents and adjoining region). For further asymmetric scenarios we refer to Zeiler et al. (2010).

We first show that in this model even very little interaction leads to profound structural changes in the optimal solutions. Second, if the system starts in a certain area of the state space, the optimal outcome reacts very sensitively to the degree of interaction. Third, when one system is uncontrollable, the outcome is determined, but interaction affects substantially the optimal paths that lead there. 


\subsection{Optimal Control of Uncoupled, Jointly Managed Systems}

Figure $1 i$ shows a classic DNSS structure in a one-dimensional system. The candidates for optimality are the stable manifolds of the saddles $\underline{A}$ and $\bar{A}$ (the direction of the phase flows is indicated by arrows). Around the unstable, central focus-equilibrium the stable manifolds overlap. By calculating the costs of a solution starting on either of these manifolds the point of equity is found that indicates the DNSS point $A_{D N S S}$ (vertical line).

Figure 1ii shows the optimal solution of two parallel, decoupled systems that are jointly managed. Without interaction the optimal outcome depends on the initial value of each state only. If either of the states exceeds (falls below) $A_{D N S S}=B_{D N S S}$, that state converges to the corresponding high (low) steady-state value. Since each of the two states can independently approach either of two steady states, this results in four optimal steady states that are separated by straight DNSS lines intersecting at the point $\left(A_{D N S S}, A_{D N S S}\right)$.

If the system starts on the vertical DNSS line, for example at $\left(A_{D N S S}, B_{0}\right)$ with some $B_{0}>A_{D N S S}, B$ converges independently of $A$ to the high level of use, whereas for $A$ there are two distinct optimal controls that drive this state to either $\underline{A}$ or $\bar{A}$ while incurring equal costs. The central DNSS point, $\left(A_{D N S S}, A_{D N S S}\right)$ is a trivial quadruple DNSS point, at which the decision maker has four different options to choose from.

Here and in the following the steady states are denoted by $(L, L),(H, L)$, $(L, H)$ and $(H, H) . L$ (low) and $H$ (high) are descriptions of the location of the respective steady state coordinate and have to be seen relatively, not in absolute $A$ or $B$ values.

\subsection{Interaction Leads to Global Changes in the Optimal Solutions}

Here the two separate state dynamics are identical, but there is now a constant per capita flow at rate $\gamma$ from each state to the other. In practice the flow rates out of the two states may be different, but we make them identical to show that the interesting results below do not depend on asymmetry in those flow rates. We analyze the changes of the optimal solutions on a global level by applying bifurcation theory.

To begin with we may anticipate some results for identically parameterized, connected systems from the uncoupled case. First, since the connecting flows annihilate each other when identical, the overall high and low level of use are not affected by growing interaction. Second, for all unique solutions that start on the 45 degree line, i.e. all solutions for $\left(A_{0}, B_{0}\right)$ with $A_{0}=B_{0} \neq A_{D N S S}$ the optimal solutions are invariant to changes of $\gamma$. Thus, 

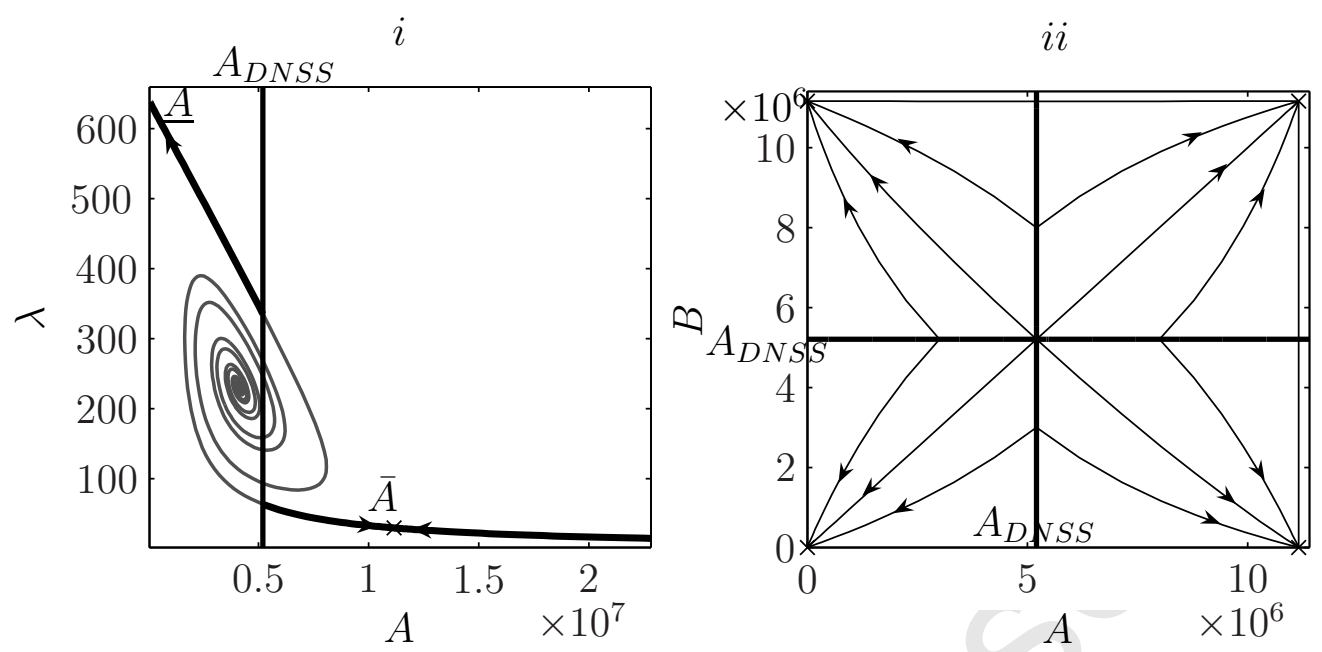

Figure 1: Phase portrait of the one-state one-control optimal control problem $(i)$ and of two uncoupled systems $(i i)$.

this central line represents the projection of the one-dimensional case into the two-dimensional system and is comprised of trajectories that appear in all later discussed cases.

Figure 2 shows phase portraits for four different levels of the interaction parameter $\gamma$ in the $A B$-plane; (i) $\gamma=0.001$, (ii) $\gamma=0.005$, (iii) $\gamma=0.01$, and $(i v) \gamma=1$. (These should be construed as rates of flow per year, because the time scale underpinning the rest of the parametrization is that $t=1$ is one year.)

In this series we can observe two structural changes. First, the number of steady states reduces from four to two between case (iii) and (iv). Second, at some point between (ii) and (iii) the asymmetric steady states (denoted by $(L, H)$ and $(H, L))$ lose optimality. This causes the disappearance of the two corresponding DNSS thresholds that separated these steady states from $(L, L)$ and $(H, H)$. The first change is a local bifurcation, precisely a fold bifurcation, that does not directly affect the optimal vector field and is hence of minor interest.

However, the loss of optimality of the asymmetric steady states is a global phenomenon that indicates a qualitative change between the systems. At this point the entire structure of the optimal trajectories is changed. Keeping one state small while the other is large is (for some initial values) optimal when interaction is low, but such a discordant policy cannot be optimal when interaction is larger than a certain critical value. The exact bifurcation 
(i) $\gamma=0.001$

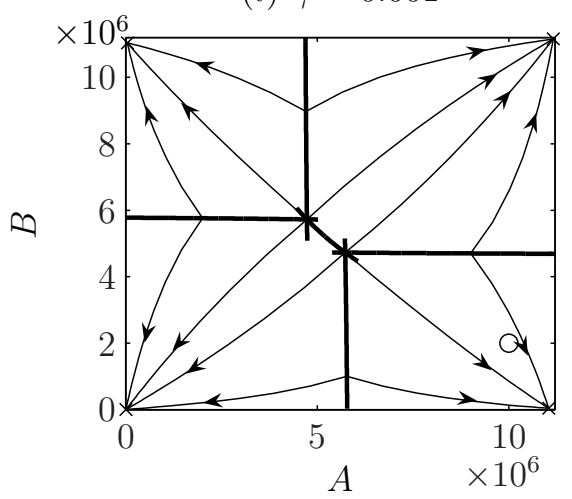

(iii) $\gamma=0.01$

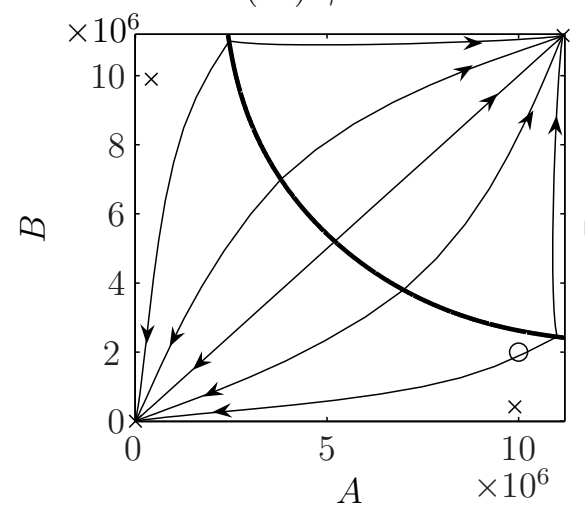

(ii) $\gamma=0.005$

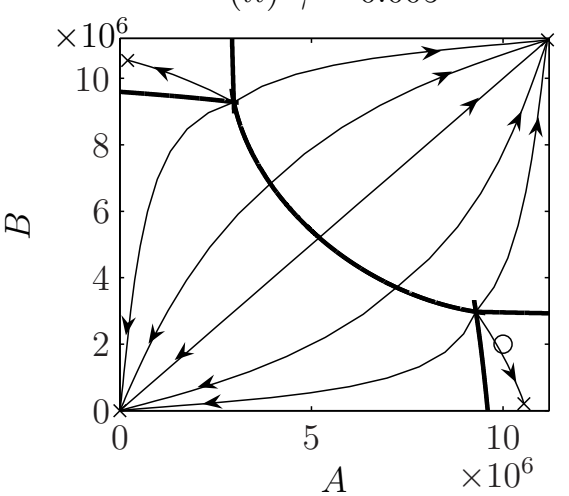

(iv) $\gamma=1$

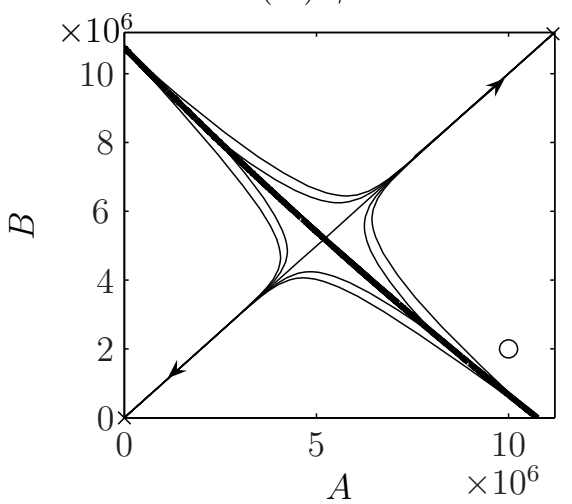

Figure 2: Phase portraits of identical epidemics for different levels of interaction, $(i)$ $\gamma=0.001$, $($ ii $) \gamma=0.005$, (iii) $\gamma=0.01$, and $(i v) \gamma=1$. Bold lines are DNSS curves, thin lines are optimal trajectories for given initial values on the DNSS curves. Steady states are indicated by an " $\times$ "; " " indicates a particular initial value, $P$. The direction of the phase flow is indicated by arrows. 
value represents an economic hairline case; its practical relevance is little. However, the magnitude of this value compared to all other parameters is of interest, because it shows how much interaction it takes to reduce policy prescription down to eradication versus accommodation of both epidemics. We use bifurcation theory to find this threshold.

Also, Wagener (2003) recognized in one-dimensional systems a connection between the existence of DNSS thresholds and bifurcation theory. He proved for one-dimensional optimal control problems that the region in the parameter space in which a DNSS threshold exists, is bounded by the occurrence of heteroclinic bifurcations. We show numerically that this result holds here as well.

A heteroclinic bifurcation is indicated by the existence of a heteroclinic connection between two equilibria. Such a connection is an orbit that lies in the stable manifold of one, and in the unstable manifold of the other equilibrium. That is, when the system starts on such a curve, it converges for $t \rightarrow \infty$ to one steady state and for $t \rightarrow-\infty$ to the other.

With baseline parameterizations a connecting orbit occurs when $\gamma$ is close to $\gamma_{\text {crit }}=0.0051987$. Figure 3 shows this connection in the state-costate space. When the system starts in a neighborhood of the asymmetric steady state it converges to $(L, L)$, i.e. the stable manifold of $(L, L)$ (that gives the optimal solutions) is in the unstable manifold of $(L, H)$.

Figure 4 highlights how the DNSS threshold "disappears" at the bifurcation. It shows the Hamiltonian calculated for initial values along the intersection of the state space that is the direct connection between $(L, L)$ and $(L, H)$. The solid line gives the costs when the system starts at any given point on this intersection and converges to $(L, L)$; the dashed line shows the costs for converging to $(L, H) .(L, L)$ is the optimal choice for all initial values with $A<\hat{A}_{(L, H)}$ since here $\mathcal{H}_{(L, L)}<\mathcal{H}_{(L, H)}$, but in $\hat{A}_{(L, H)}$ the Hamiltonians coincide, so this steady state is a weak DNSS point (cf. Grass et al., 2008).

In this case of a weak DNSS point, the optimal solution is unique for each initial point, but depending on the initial state the long-run optimal solution may be different. In other words, weak DNSS points only exhibit the property of history dependence not that of multiplicity of the optimal solution. Here, when starting in $(L, H)$, the system stays in $(L, H)$, as $(L, H)$ is a steady state that is not dominated by a different path (the values of the Hamiltonians are equal). So, the optimal solution is unique. However, small deviations of the initial values cause the system instead to tip to a different 
steady state, i.e., to converge to $(L, L)$.

From the existence of the heteroclinic connection in the two-dimensional model we can draw implications both methodologically and substantively. Methodologically, our model illustrates that Wagener's general result on the existence of DNSS thresholds in one-dimensional models applies in this case to a two dimensional model.

Another interesting methodological finding is depicted in Figure 5, which shows the phase portrait for a value of $\gamma$ that is only slightly larger (the difference is less than $\left.10^{-7}\right)$ than its bifurcation value. The $(L, H)-(L, L)$ DNSS curve does not just disappear but is replaced by an $(L, L)$-indifference curve that separates two different paths, e.g. $p t_{1}$ and $p t_{2}$, that both approach $(L, L)$ while incurring equal costs. Since very little is known on what exactly happens when a two-dimensional DNSS threshold disappears, this phenomenon raises questions, including whether DNSS curves are in general replaced by such indifference curves.

Substantively the important finding is that the bifurcation value $\gamma_{\text {crit }}=$ 0.0051987 is surprisingly low. When comparing it to the rate of natural quitting, $\mu p^{b}=0.107692, \gamma_{\text {crit }}$ is about one twentieth as large. So, even a very little migration is sufficient to achieve substantial changes in the optimal policy.

\subsection{Changes of the Optimal Outcome are Non-Monotonic in the Strength of the Interaction}

In the last section we saw that small levels of interaction between identical systems can lead to structural changes in the optimal solution. Here, we go to a more local level and present an example where the optimal policy for a certain initial value changes dramatically when the degree of interaction is varied. Furthermore, we discover a non-monotonicity of the optimal policy in the strength of interaction. One might expect that growing interaction favors either eradication or it favors accommodation, but we see that this is not the case.

We consider the initial value $P=(10,2)$ million, which corresponds to a well established population $A$ that is close to its steady state value and a second population, $B$, that is on the verge of spreading out, perhaps because the substance has only recently become available to this population. The changes are illustrated with the cases depicted in Figure 2 where $P$ is indicated by an "o"; the respective trajectories are shown in Figure 6 . 

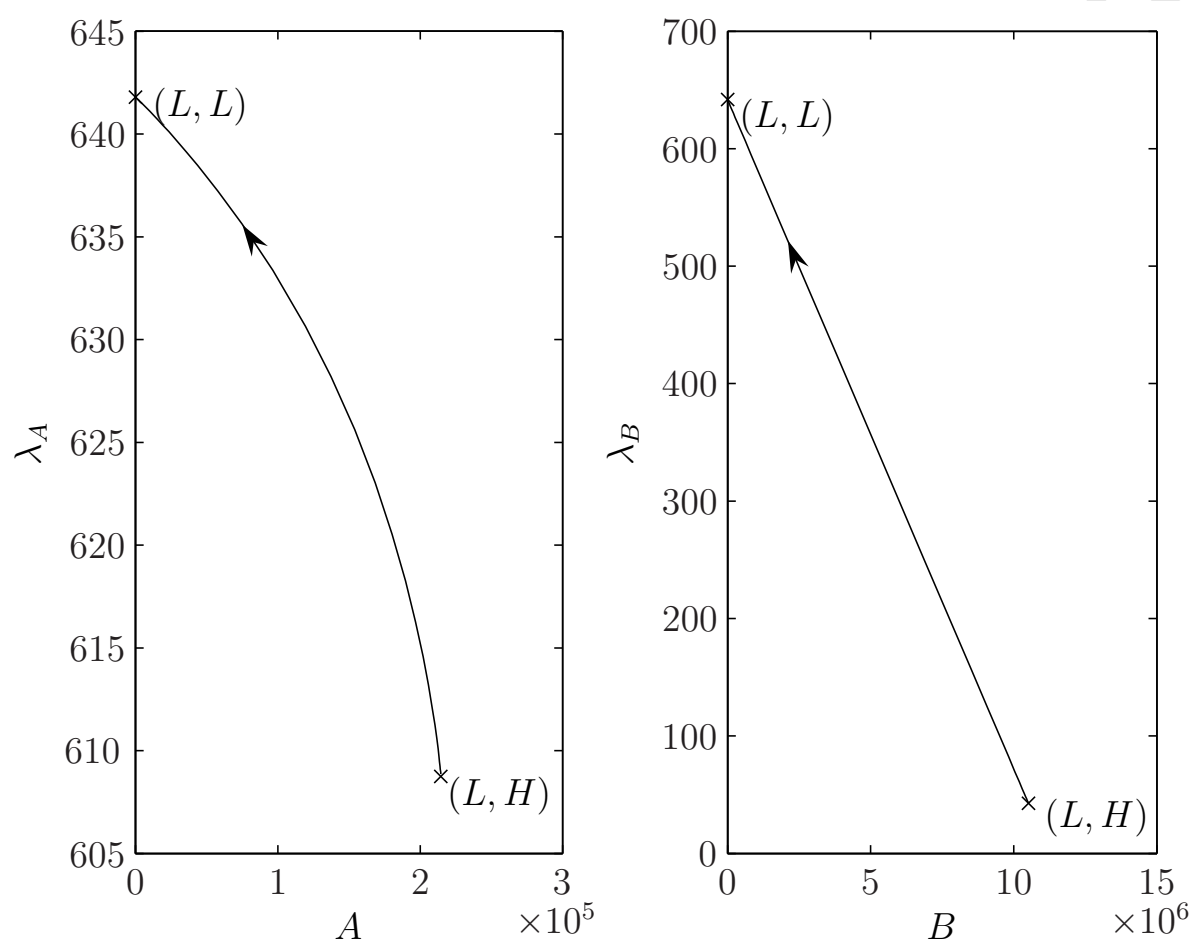

Figure 3: Heteroclinic connection of two equilibria at $\gamma_{c r i t}=0.0051987$. 


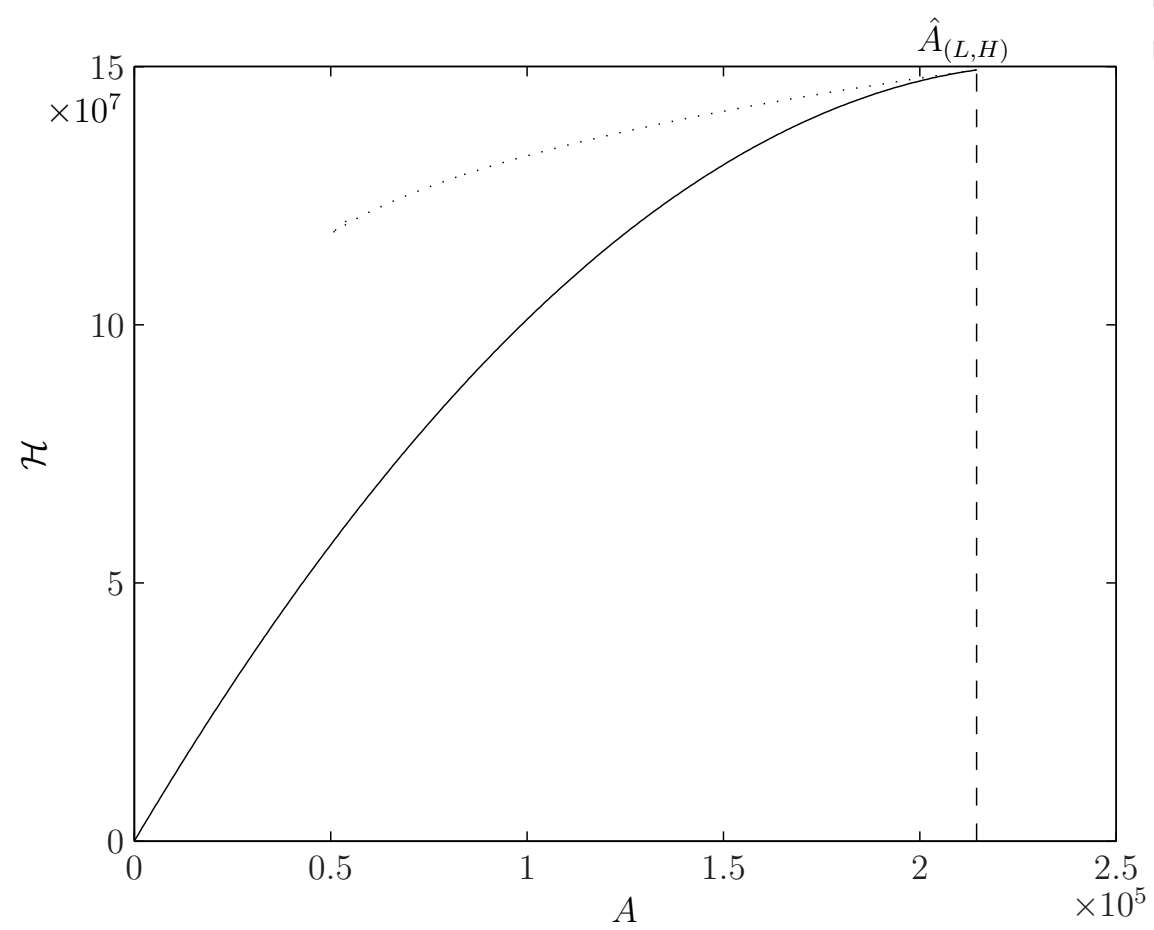

Figure 4: Values of the Hamiltonian as a function of $A$. On the solid line the low level of use is approached; on the dashed line $(L, H)$ is approached. The values of the Hamiltonian coincide in $(L, H)$. 


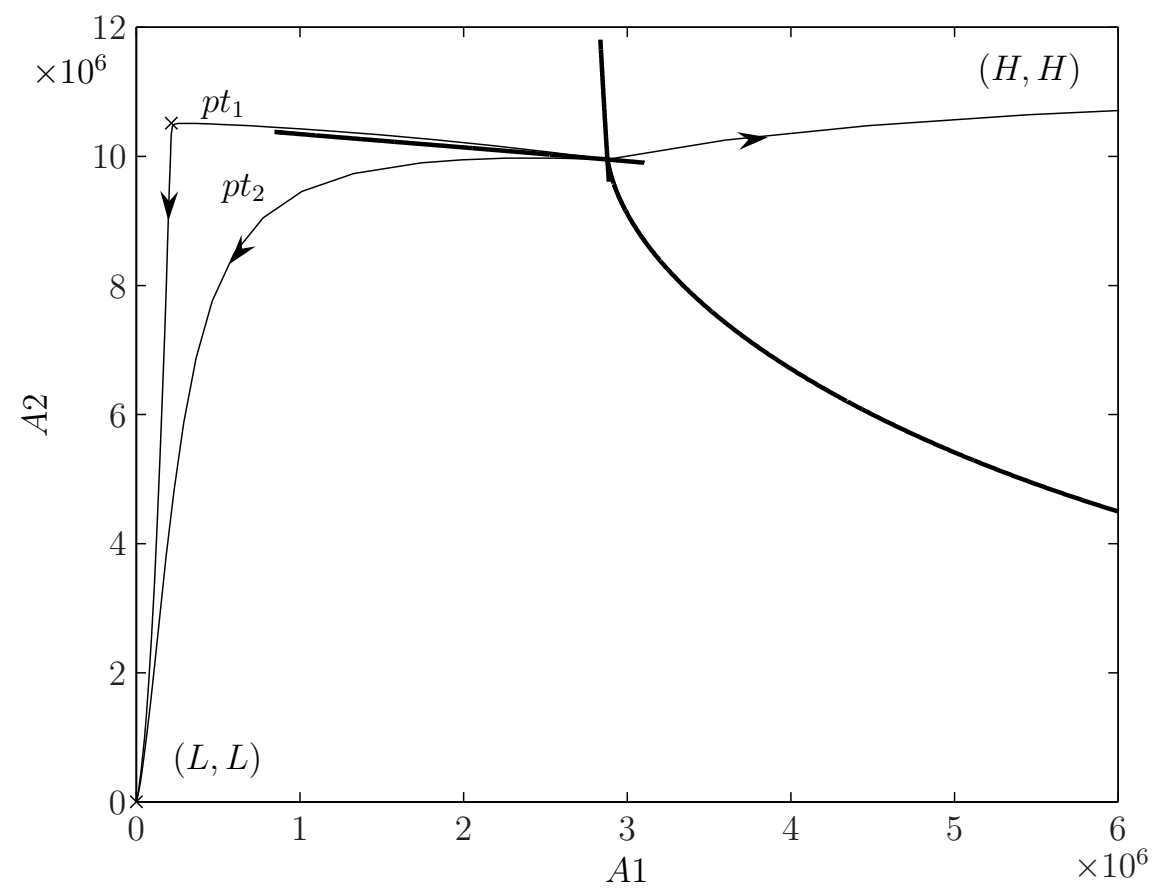

Figure 5: Identical epidemics at the heteroclinic bifurcation value of $\gamma, \gamma_{c r i t}=0.0051987$. 


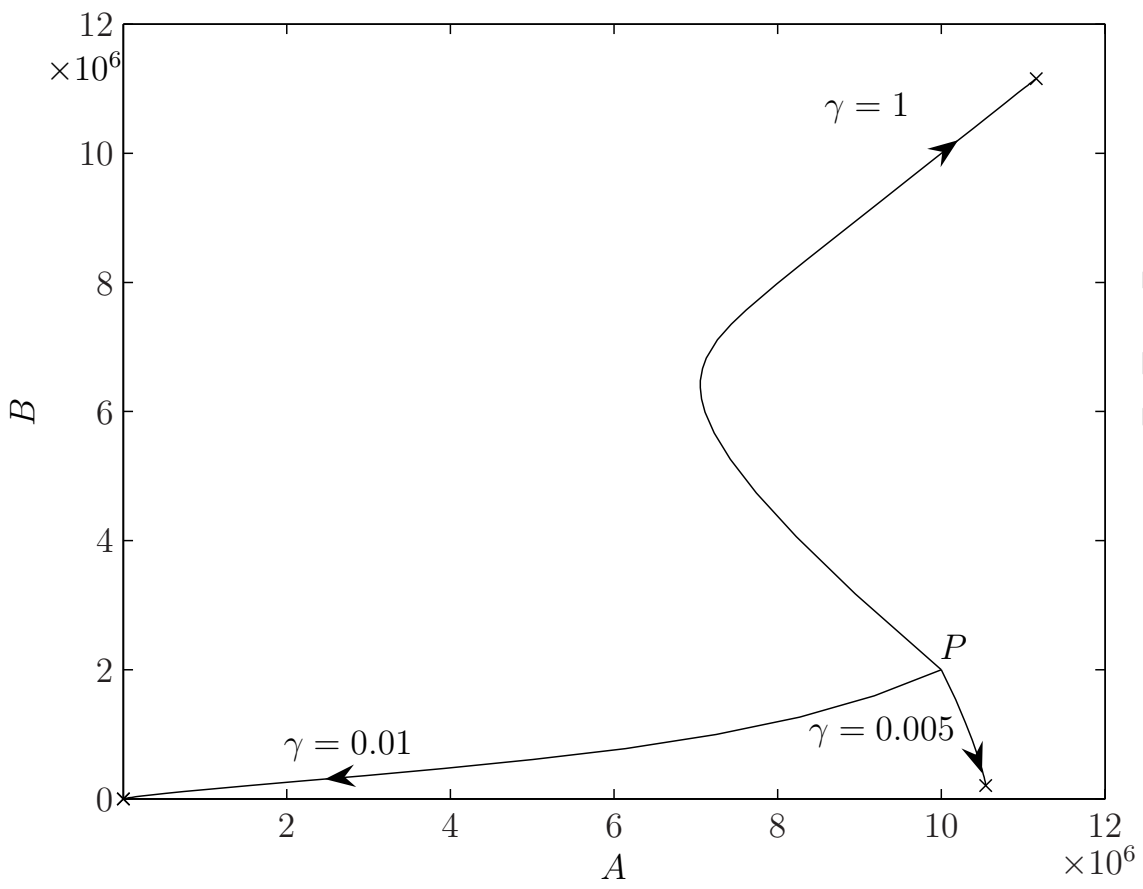

Figure 6: Optimal paths for different levels of interaction, all starting at a certain initial value, $P$.

We can distinguish between three levels of interaction, $\gamma\left\langle\gamma_{\text {crit }}, \gamma\right\rangle \gamma_{\text {crit }}$ and $\gamma>>\gamma_{\text {crit }}$.

$\gamma<\gamma_{\text {crit }}$ For small levels of interaction the system adheres to the basic structure of four optimal steady states and converges for $P$ to $(H, L)$ (Figures $2 i$ and $i i$ and path $\gamma=0.005$ in Figure 6 ). The major population grows to its steady state level, while the smaller population is "eradicated". With growing interaction (e.g. from $\gamma=0.001$ to $\gamma=0.005$ ) the basins of attraction of the asymmetric steady states get smaller, because maintenance of the smaller epidemic at a low level gets harder with the stronger inflow from the larger epidemic.

$\gamma>\gamma_{\text {crit }}$ When interaction is just a little larger than the critical value derived in the last section the asymmetric steady states lose optimality, so we know that strategy is no longer optimal. $P$ lies then in the basin of attraction of $(L, L)$ and for both epidemics eradication is optimal 
(Figure 2iii, $\gamma=0.01$ in Figure 6). That seems to be counterintuitive because a larger interaction leads to a stronger bond between the epidemics and one could assume that the larger state drags the smaller to a high steady state.

A possible explanation is that without control, $A$ and $B$ would converge to the high level of use. Even in a situation where there are initially no users in $B, A$ would cause a persistent drug problem $B$. Optimal control may prevent this. So even if $\operatorname{drug} A$ is past the (single) drug threshold where it is optimal to accommodate, the prevention of the emergence of a second drug problem can suddenly make it optimal to switch to an eradication strategy not only for $\operatorname{drug} B$ but also for drug $A$. This holds for a relatively moderate linkage of the systems.

$\gamma>>\gamma_{c r i t}$ With a further increase of interaction the situation changes again. Connecting flows of this scale $(\gamma=1$ and larger $)$ tie the systems so closely together that the optimal trajectories of $A$ and $B$ coincide after a short period of time. In the phase portrait (Figure 2iv) this manifests in the trajectories moving close to the 45 degree line.

The convexity of the DNSS curve that separates $(L, L)$ from $(H, H)$ lessens, thus the basin of attraction of $(H, H)$ expands and the basin of $(L, L)$ shrinks. As a consequence, the optimal policy for $P$ changes yet again, this time from eradication to accommodation of both drugs. When the states are so strongly connected it is only the sum of the states that determines the optimal outcome. The DNSS curve is close to $A+B=2 A_{D N S S}$, where $A_{D N S S}$ is the DNSS threshold of the corresponding one-dimensional system. In this case the two-dimensional system is merely the sum of the two one-dimensional systems and may be treated as a one-dimensional system.

This example shows that even small perturbations of the linkage of the systems can lead to a total reversal of the optimal policy. The non-monotonicity of the optimal policy with respect to the nature of the linkage of the systems underlines the importance of the potential impact of a second, parallel system. Since for certain initial values a small change or misunderstanding of the strength of interaction can change the optimal policy as profoundly as from eradication to accommodation, a very good understanding of that linkage is crucial. 
One comment is in order. The central DNSS curve is convex in the states. That implies that for moderate levels of interaction the optimal long-run steady state depends on the relation and not only the sum of $A(0)$ to $B(0)$. The greater the differences are between $A(0)$ and $B(0)$, the more favorable is an eradication strategy. From an application point of view, this is a sensible result. Small epidemics are more easily controlled (cf. the marginal efficiency of control), so the decision maker can then concentrate on the larger epidemic, where a reduction of the number of users has (because of the nature of the interaction) a greater impact on the system.

\subsection{In Two-Dimensional Systems Not All Thresholds are Tipping Points}

So far we have discussed two identical systems that could be independently controlled. But what if a parallel system exists that is outside the influence of the decision maker? In the Ramsey growth model this would be a large economy in a neighboring country. For the control of fashion we could imagine a competing product that emerges. And in illicit drug use it could be drug users in an adjacent country that do not impose costs on the decision maker but interact by migration with the decision maker's drug problem.

Hence, in the following we discuss a case where parallel systems are symmetrically connected and only one of the systems, $A$, is controllable and costly to the decision maker, i.e. for the social costs parameter we have $\kappa_{B}=0$ and for the control, the efficiency of $u_{B}$ is $c_{B}=0$.

In such a situation two unconnected systems would yield three optimal steady states. The uncontrolled system converges for all initial values to a high steady state, whereas the controlled system converges to either of two locally optimal equilibria that are separated by a DNSS threshold. By introducing interaction with $B>0$ a persistent flow of users from $B$ to $A$ exists that makes it difficult to preserve an overall low level of use. The level of interaction we have chosen is $\gamma=0.01$. That is high enough that in Figure 2 the structure of four optimal steady states is not optimal anymore.

Figure 7 shows a phase portrait in the state space. There is only a single optimal steady state, where both epidemics are at a high level of use. Consequently there is no DNSS curve. However, the influence of the parallel system manifests in the occurrence of an "indifference" curve that separates two different optimal policies that lead to the same steady state. So, not only is the optimal outcome influenced but so is the way the system gets there. The same phenomenon occurred in the hairline case depicted in Figure 5, 
however the indifference curve here exists in a sufficiently large region of the parameter space to qualify as relevant for practical applications.

When the system starts at a point on the indifference curve, such as $(A, B)=(2.1,0.2)$, the decision maker can choose between optimal control strategies that result in either trajectory $p t_{1}$ or $p t_{2}$. Either strategy incurs the same total costs. The number of costly users, $A$, is for the most part significantly lower on $p t_{1}$ than on $p t_{2}$, but the control costs are higher which is reflected in the optimal controls along these paths, shown in Figure 7.

When choosing $p t_{1}$, the decision maker applies $u_{A}^{p t_{1}}$ to a great extent, thus driving the number of users in $A$ down at first. After passing this point, little control is applied and the epidemics grow to the steady state value. The sharp bend of $u_{A}^{p t_{1}}$ is due to the projection of the control into the $A$ - $u$ space. In the phase portrait the optimal path stays at a very low $A$-level for some time which cannot be shown in the projection. Along path $p t_{2}$ the epidemics head directly to the steady state, driven by control $u_{A}^{p t_{2}}$, which stays at a moderate level throughout the course of the epidemic. The money saved by using a weaker control is offset by the larger number of users in the $A$ state.

Hence, what we observe here is a point of indifference that does not separate the basins of attraction of different equilibria, but rather separates dramatically different strategies for getting to a single equilibrium.

This cannot happen in isolated one-dimensional systems, for which such indifference points can be excluded. The present example shows that in interacting systems answering "where should we go?" is not sufficient. One must also ask, "Even given where we should go, which among multiple optimal ways of getting there should we choose?". Hence, looking only at the isolated one-dimensional system may simplify to the point of distortion even the way the choices are framed. So the discussion has to become more specific and the "dimension" of the questions asked has to be adapted to fit the complexity of the system considered.

\section{Discussion and Conclusions}

In this paper we generalized a one-dimensional optimal control problem with a DNSS property to a two-dimensional optimal control problem by taking the "direct product" of the model with itself. The resulting problem describes a situation where two similar, parallel systems are jointly managed by a single decision maker. 

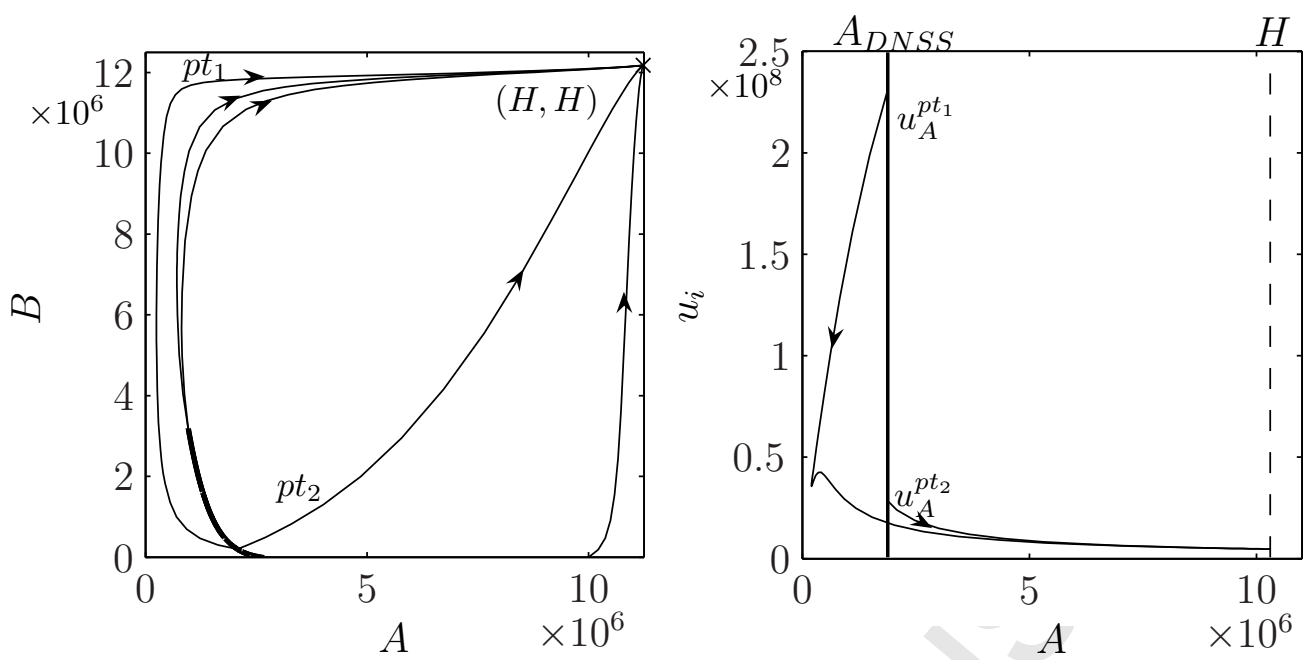

Figure 7: Similar drugs in different regions, $\kappa_{B}=0, c_{B}=0$ and $\gamma=0.01$. The left figure shows a phase portrait with the "indifference" curve highlighted. The right figure shows the optimal controls along the paths starting on the indifference curve on $(A, B)=(2.1,0.2)$.

We use a particular example out of the domain of drug control to perform a case study on this class of models. The model is constructed in a way that it easily can be adapted to fit a whole range of relevant scenarios; for a deeper discussion see Zeiler (2007). We start the analysis with two identically parameterized models and show by gradually introducing interaction that a little interaction can have a tremendous impact on the optimal solution. In fact, a minor change can actually reverse the policy prescription yielded from the analysis of an underlying one-dimensional system.

This finding is all the more significant when one considers that not just the magnitude of the interaction was modest, but also that the nature of the interaction belongs to a specific class, namely the migration from one system to the other with no net creation or destruction of the sum of the state variables in total caused (directly) by interaction of the two states. That is, the sum $\dot{x}+\dot{y}$ depends only on $(x, y)$ and not at all on the presence or extent of interaction.

Furthermore, we could show that some general invariance properties one might have expected to hold, do not in fact hold. For example, one might have expected that if in an already escalated situation a high level of drug use is optimal in the one-state model, that this carries over to the twodimensional coupled case. However, we show that this does not necessarily 
hold true, because the optimal outcome depends non-monotonically on interaction. What is optimal for small interaction may be optimal for strong interaction, but not for an intermediate situation.

Another important finding in the drug control context is that the well established paradigm of "eradication" or "accommodation" has to be reconsidered. When two interacting drug markets are modeled, the optimal answer may be a mix of "eradication" and "accommodation" strategies, the optimality of which can crucially depend on the initial state of the system. So, in further analyses the whole system of drug use should be taken into account. So researchers could take this as inspiration for finding out more about the nature of linkages between substances of abuse.

The analysis performed here may be specific for drug control, however, our approach can be carried over to other optimal control problems that concern other domains. We presented here a proof by example that introducing modest interaction into the product of two isolated one-state systems can make a big difference. This suggests that a sensible line of further research would be to revisit various classic one-dimensional systems and pursue parallel analyses in those other domains as well.

A generalization of Sethi's (1979) optimal advertising problem to two states for example, could reveal surprising results on advertising different, exchangeable products (e.g. two cars of the same automaker). The optimal advertising policy may be changed by the introduction of a new product. The question of whether to advertise or not may have to be extended by the second dimension to discordant strategies and the answer might depend crucially on the degree of interaction (i.e. the change of the product preferred by customers).

A scenario akin to the uncontrollable drug problem in an adjacent country (Section 3.4) can arise in a higher dimensional version of the Ramsey growth model. If there exists a second economy sufficiently large that it does not act strategically with respect to the first economy then this parallel, interacting system may have a large impact on the optimal strategy of the first country, even if the second economy does not play a direct role in the objective function. This setting could be studied in a game theoretic framework, as well. Here, the uncontrolled state could be controlled by another player who is influenced by the behavior of the focal player. DNSS thresholds in such a setting would enrich existing literature on this phenomenon (cf. Dechert and O'Donnell, 2006).

Further models for possible investigation include a higher dimensional 
version of the shallow lake system investigated by e.g. Mäler et al. (2003), multi-dimensional models in the area of other economic problems, such as multiple capital stocks, and models of infectious disease dynamics.

Besides substantively interesting insights we found two methodological implications as well. First, the occurrence of the heteroclinic connection in our model that bounds the area in the parameter space where DNSS thresholds exist hints that Wagener's general result on the existence of DNSS thresholds in one-dimensional models might extend to higher dimensional models. This is relevant, because analytically results on DNSS thresholds are rare in the literature.

Second, by introducing some asymmetry to the model we could find an "indifference" curve of significant length. Although this phenomenon is not new in optimal control theory, it is rare and important enough to be highlighted here. In a one-dimensional model, tipping point behavior is restricted to DNSS points, starting at which there are two options, namely approaching a low-level steady state or a high-level steady state. In two-dimensional models, however, "low" or "high" are not the only choices, so the paths leading to an optimal outcome, can become a policy relevant question.

In conclusion, we set out to address the question "Can the presence of an additional, parallel one-state system substantially impact the policy prescriptions for the original one-state system?". We know now that the simple answer is "Yes, and it can do so in several ways."

\section{Acknowledgement}

This work was supported by the Austrian Science Fund (FWF) under contract No. P18527-G14, the Qatar National Research Fund, and by a Robert Wood Johnson Foundation Investigator Award in Health Policy Research. The views expressed imply no endorsement by the Robert Wood Johnson Foundation.

The authors gratefully acknowledge the helpful and inspiring comments of Gustav Feichtinger, Dieter Grass, Peter Kort, Alois Steindl, and of one anonymous reviewer.

\section{References}

Behrens, D. A., Caulkins, J. P., Tragler, G., Feichtinger, G., 2000. Optimal control of drug epidemics: Prevent and treat - but not at the same time? Management Science 46, 333-347. 
Behrens, D. A., Caulkins, J. P., Tragler, G., Feichtinger, G., 2002. Why present-oriented societies undergo cycles of drug epidemics. Journal of Economic Dynamics and Control 26, 919-936.

Brock, W. A., Dechert, W. D., 1983. The Generalized Maximum Principle. Tech. Rep. 8316, Social Science Research Institute.

Brock, W. A., Starret, D., 2003. Managing systems with nonconvex positive feedback. Environmental and Resource Economics 26, 575-602.

Bultmann, R., Caulkins, J. P., Feichtinger, G., Tragler, G., 2008a. How should drug policy respond to market disruptions? Contemporary Drug Problems 35, 371-395.

Bultmann, R., Caulkins, J. P., Feichtinger, G., Tragler, G., 2008b. Modeling supply shocks in optimal control models of illicit drug consumption. In: Lirkov, I., Margenov, S., and Wasniewski, J. (Ed.). Lecture Notes in Computer Sciences Vol. 4818. Springer, Heidelberg, 338-367.

Caulkins, J. P., 2008. Implications of inertia for assessing drug control policy. Contemporary Drug Problems 35, 347-369.

Caulkins, J. P., Feichtinger, G., Johnson, M., Tragler, G., Yegorov, Y., 2005. Skiba thresholds in a model of controlled migration. Journal of Economic Behavior \& Organization 57, 490-508.

Caulkins, J. P., Hartl, R. F., Kort, P. M., Feichtinger, G., 2007. Explaining fashion cycles: Imitators chasing innovators in product space. Journal of Economic Dynamics and Control 31, 1535-1556.

Dechert, W. D., Nishimura, K., 1983. A complete characterization of optimal growth paths in an aggregated model with a non-concave production function. Journal of Economic Theory 31, 332-354.

Dechert, W. D., O’Donnell, S., 2006. The stochastic lake game: A numerical solution. Journal of Economic Dynamics \& Control 30, 1569-1587.

Feichtinger, G., Grienauer, W., Tragler, G., 2002. Optimal dynamic law enforcement. European Journal of Operational Research 141, 58-69. 
Feichtinger, G., Hartl, R. F., 1986. Optimale Kontrolle ökonomischer Prozesse - Anwendungen des Maximumsprinzips in den Wirtschaftswissenschaften. Walter de Gruyter, Berlin.

Ferrence, R., 2001. Diffusion theory and drug use. Addiction 96, 165-173.

Gould, J., 1970. Diffusion processes and optimal advertising policy. In: Phelps, E.S. (Ed.). Microeconomic Foundations of Employment and Inflation Theory. W.W. Norton, New York, 338-367.

Grass, D., Caulkins, J. P., Feichtinger, G., Tragler, G., Behrens, D., 2008. Optimal Control of Nonlinear Processes: With Applications in Drugs, Corruption and Terror. Springer, Heidelberg.

Kleiman, M. A. R., 1993. Enforcement swamping: A positive-feedback mechanism in rates of illicit activity. Mathematical and Computer Modelling 17, $65-75$.

Leonard, D., Long, N., 1992. Optimal Control Theory and Static Optimization in Economics. Cambridge University Press, Cambridge.

Mäler, K. G., Xepapadeas, A., de Zeeuw, A., 2003. The economics of shallow lakes. Environmental and Resource Economics 26, 603-624.

Rachlis, B., Brouwer, K. C., Mills, E. J., Hayes, M., Kerr, T., Hogg, R. S., 2007. Migration and transmission of blood-borne infections among injection drug users: Understanding the epidemiologic bridge. Drug and Alcohol Dependence 90, 107-119.

Roxburgh, A., Degenhardt, L., Breen, C., 2004. Changes in patterns of drug use among injecting drug users following changes in the availability of heroin in New South Wales, Australia. Drug and Alcohol Review 23, 287294.

Rydell, C. P., Caulkins, J. P., Everingham, S. S., 1996. Enforcement or treatment: Modeling the relative efficacy of alternatives for controlling cocaine. Operations Research 44, 687-695.

Schelling, T., 1967. Economic analysis of organized crime. In: President's Comission on Law Enforcement and the Administration of Justice Task Force. Washington, D.C.: Government Printing Office. 
Schelling, T., 1973. Hockey helmets, concealed weapons, and daylight saving: A study of binary choices with externalities. Journal of Conflict Resolution $17,381-428$.

Sethi, S., 1977. Nearest feasible paths in optimal control problems: Theory, examples, and counterexamples. Journal of Optimization Theory and Applications 23, 563-579.

Sethi, S., 1979. Optimal advertising policy with the contagion model. Journal of Optimization Theory and Applications 29, 615-627.

Skiba, A. K., 1978. Optimal growth with a convex-concave production function. Econometrica 46, 527-539.

Tragler, G., Caulkins, J. P., Feichtinger, G., 2001. Optimal dynamic allocation of treatment and enforcement in illicit drug control. Operations Research 49, 352-362.

Wagener, F., 2003. Skiba points and heteroclinic bifurcations, with applications to the shallow lake system. Journal of Economic Dynamics and Control 27, 1533-1561.

Zeiler, I., 2007. Optimal dynamic control with DNSS curves: Multiple equilibria in epidemic models of HIV/AIDS and illicit drug use. Ph.d. thesis, Vienna University of Technology, Faculty of Mathematics and Geoinformation, Vienna, Austria.

Zeiler, I., Caulkins, J. P., Tragler, G., 2010. When two become one: Optimal control of interacting drug epidemics. Research Report 2010-07, Institute for Mathematical Methods in Economics, TU Wien. 